American Journal of Biostatistics 1 (2): 82-93, 2010

ISSN 1948-9889

(C) 2010 Science Publications

\title{
Some Test Statistics for Testing the Binomial Parameter: Empirical Power Comparison
}

\author{
Florence George and B.M. Golam Kibria \\ Department of Mathematics and Statistics, \\ Florida International University FIU, Miami, FL 33193, USA
}

\begin{abstract}
Problem statement: The Binomial distribution is one of the most useful probability distributions in the filed of quality control, physical and medical sceinces. Many questions of interest to the health worker related to make inference about the unknown population proportion, parameter of binomial distribution. This study considers the problem of hypotheses testing of the parameter of a binomial distribution. Approach: Different test statistics available in literature are reviewed and compared based on the empirical size and power properties. Since a theoretical comparison is not possible, a simulation study has been conducted to compare the performance of the test statistics. To illustrate the findings of the paper, two real life health related data are analyzed. Results: The simulation study suggests that some methods have better size and power properties than the other test statistics. The performnace of the proposed test statistics also depend on the hypothesized value of the binomial parameter. Conclusions/Recommendations: The practitioners should be careful about the hypothesized value of the binomial parameter $\mathrm{p}$. If the hypothesized value is near 0.5 , any test is acceptable for moderate to large sample size. However, for testing the end or small value of $p$, one might need very large sample size to have a good power and actual size of the test.
\end{abstract}

Key words: Binomial distribution, empirical power, hypothesis testing, simulation study, Type I error, AMS 2000 subject classifications, primary 62F03, secondary 62F40, binomial distribution, fasting glucose, binomial proportion, null hypothesis

\section{INTRODUCTION}

The Binomial distribution is one of the most widely encountered probability distributions in applied statistics. Many question of interest to the health worker relate to make inference about the unknown population proportion, parameter of binomial distribution. For example, one may be interested in the proportion of recovery out of patients receiving a particular treatment or percentage of subjects with Impaired fasting Glucose in a population of interest (Wagenknecht et al., 2003). To make inference about the unknown parameter of the binomial dis-tribution, one may consider either confidence interval or hypothesis testing. Several authors in several times have discussed the confidence interval estimates. Among them, Agresti and Coull (1998), Anscombe (1948), Barker (2002), Bartlett (1947), Cai (2005), Casella (1986), Efron (1987), Flowerdew and Aitkin ( 2006), Freeman and Tukey 1950), Garwood (1936), Wilson (1927) are notable. However, the lit-erature on the test statistics for testing the proportion of success of a binomial distribution is limited. This study made an attempt to consider various available test statistics, namely, exact Clopper Pearson Method, Bayesian Method, Wilson method without continuity, Wilson method with continuity correction, Wald method without continuity, Wald method with continuity, recentered Wald without continuity, recentered Wald with continuity, Bootstrap 1method without continuity, Bootstrap method with continuity correction, arcsine (variance stabilizing) transformation, arcsine (variance stabilizing) transformation with a continuity correction for testing the binomial proportion and compare them under the same simulation conditions. Therefore, the important contribution of this study is to compare several test statistics proposed by several researchers in several times under the same simulation condition and to find some good test statistics based on size and power of the test. Since a theoretical comparison is not possible, a simulation study has been made to compare performances of the proposed test statistics.

Corresponding Author: Florence George, Department of Mathematics and Statistics, Florida International University FIU, Miami, FL 33193, USA 
Am. J. Biostatistics 1 (2): 82-93, 2010

\section{METERIALS AND METHODS}

Suppose $X_{1}, X_{2}, X_{n}$ be a iid random sample from a binomial population with parameters $n$ and $p$. Consider the following hypothesis:

Null hypothesis: $\mathrm{H}_{0}: \mathrm{p}=\mathrm{p}_{0}$

Alternative hypothesis: $\mathrm{H}_{\mathrm{a}}: \mathrm{p}=\mathrm{p}_{0} \pm \mathrm{c}$

Where $c \in(0,1)$ is a positive constant. Here we are interested for a two tailed test. However, one may easily follow the same procedure for left or right tailed tests. When $\mathrm{c}=0$, we get size of the test (type I error rate $(\alpha)$ ). When $c \neq 0$, we get powers $(1-\beta)$ of the test statistic. Our objective is to test against a proposed value of the parameter $p$ with a specific significance level. We have considered 14 different test statistics for testing the binomial parameter $\mathrm{p}$. Since the references for all proposed test statistics are available, we briefly discussed them here.

Method 1-exact clopper pearson method: Following Clopper and Pearson (1934), we reject the null hypothesis (1) at $\alpha$ level of significance when:

$\widehat{\mathrm{P}}<\operatorname{BetaX}_{0, \mathrm{n}}-\mathrm{X}_{0+1, \alpha / 2}$ or BetaX $\mathrm{X}_{0+1, \mathrm{n}}-\mathrm{X}_{0,1-\alpha / 2}$

where, $\hat{\mathrm{P}}<\mathrm{X} / \mathrm{n}$ denotes the sample proportion and $\mathrm{X}$ is the number of successes among $n$ trials. Here BetaX $_{0, \mathrm{n}}-\mathrm{X}_{0+1, \alpha / 2}$ or BetaX $\mathrm{X}_{0+1, \mathrm{n}}-\mathrm{X}_{0,1, \alpha / 2}$ are the $\mathrm{a} / 2$ and 1$\mathrm{a} / 2$ percentile points respectively of the $\operatorname{Beta}\left(\theta_{1}, \theta_{2}\right)$ distribution.

Method 2-bayesian method: If we choose the prior distribution for the binomial pa-rameter $\mathrm{p}$ as Beta $(1,1)$, then the posterior distribution of $\mathrm{p}$ given the data will have a Beta $\left(\mathrm{X}+1, \mathrm{n}_{\mathrm{X}}+1\right)$ distribution. Following Brown et al. (2001), we reject the null hypoth-esis (1) at $\alpha$ level of significance when:

$$
\widehat{\mathrm{P}}<\operatorname{BetaX}_{0+1, \mathrm{n}} \mathrm{X}_{0+1, \alpha / 2} \text { or } \widehat{\mathrm{P}}>\operatorname{BetaX}_{0+1, \mathrm{n}}-\mathrm{X}_{0,1-\alpha / 2}
$$

Method 3-Score or Wilson method without continuity correction: Following Wilson (1927), we reject the null hypothesis (1) at a level of significance when:

$$
\begin{gathered}
\hat{\mathrm{p}} \frac{2 \mathrm{X}_{0}+\mathrm{z}_{\alpha / 2}^{2}-\mathrm{z}_{\alpha / 2} \sqrt{\mathrm{z}_{\alpha / 2}^{2}+4 \mathrm{X}_{0}\left(1-\frac{\mathrm{X}_{0}}{\mathrm{n}}\right)}}{2\left(\mathrm{n}+\mathrm{z}_{\alpha / 2}^{2}\right)} \text { or } \\
\frac{2 \mathrm{X}_{0}+\mathrm{z}_{\alpha / 2}^{2}-\mathrm{z}_{\alpha / 2} \sqrt{\mathrm{z}_{\alpha / 2}^{2}+4 \mathrm{X}_{0}\left(1-\frac{\mathrm{X}_{0}}{\mathrm{n}}\right)}}{2\left(\mathrm{n}+\mathrm{z}_{\alpha / 2}^{2}\right)}
\end{gathered}
$$

Method 4-Score or Wilson method with continuity correction: We reject the null hypothesis (1) at a level of significance when:

$$
\begin{aligned}
& \hat{\mathrm{p}} \frac{2 \mathrm{X}_{0}+\mathrm{z}_{\alpha / 2}^{2}-1-\mathrm{z}_{\alpha / 2} \sqrt{\alpha(\mathrm{x})}}{2\left(\mathrm{n}+\mathrm{z}_{\alpha / 2}^{2}\right)} \text { and } \\
& \frac{2 \mathrm{X}_{0}+\mathrm{z}_{\alpha / 2}^{2}+1+\mathrm{z}_{\alpha / 2} \sqrt{\mathrm{b}(\mathrm{x})}}{2\left(\mathrm{n}+\mathrm{z}_{\alpha / 2}^{2}\right)} \\
& \mathrm{a}(\mathrm{x})=\mathrm{z}_{\alpha / 2}^{2}-\left(2+\frac{1}{\mathrm{n}}+4 \mathrm{X}_{0}-\frac{\mathrm{X}_{0}}{\mathrm{n}}+\frac{1}{\mathrm{n}}\right) \text { and } \\
& \mathrm{b}(\mathrm{x})=\mathrm{z}_{\alpha / 2}^{2}+\left(2-\frac{1}{\mathrm{n}}+4 \mathrm{X}_{0}-\frac{\mathrm{X}_{0}}{\mathrm{n}}-\frac{1}{\mathrm{n}}\right)
\end{aligned}
$$

Method 5-Normal approximation(Wald method) without continuity: Historically, this is one of the first methods for testing a parameter (Barker, 2002) of a distribution. Wald method uses the asymptotic normality of the test statistic:

$$
\mathrm{Z}=\frac{\left(\hat{\mathrm{p}}-\mathrm{p}_{0}\right)}{\sqrt{\mathrm{p}_{0}\left(1-\mathrm{p}_{0}\right) / \mathrm{n}}}
$$

At a level of significance, the null hypothesis in (1) will be rejected, when:

$$
\left|\mathrm{z}_{10}\right|=\left|\frac{\left(\hat{\mathrm{p}}-\mathrm{p}_{0}\right)}{\sqrt{\mathrm{p}_{0}\left(1-\mathrm{p}_{0}\right) / \mathrm{n}}}\right|>\mathrm{z}_{\mathrm{a} / 2}
$$

where $z_{a / 2}$ is the upper critical value of a standard normal variate. Equation 2 implies that we will reject the null hypothesis when:

$$
\begin{aligned}
& \hat{\mathrm{p}}<\mathrm{p}_{0}-\mathrm{z}_{\mathrm{a} / 2} \sqrt{\mathrm{p}_{0}\left(1-\mathrm{p}_{0}\right) / \mathrm{n}} \text { or } \\
& \hat{\mathrm{p}}<\mathrm{p}_{0}+\mathrm{z}_{\mathrm{a} / 2} \sqrt{\mathrm{p}_{0}\left(1-\mathrm{p}_{0}\right) / \mathrm{n}}
\end{aligned}
$$

Method 6-Normal approximation(Wald method) with continuity correction: We will reject the null hypothesis in (2.1) at a level of significance when:

$$
\begin{aligned}
& \hat{\mathrm{p}}<\mathrm{p}_{0}-\mathrm{z}_{\mathrm{a} / 2} \sqrt{\mathrm{p}_{0}\left(1-\mathrm{p}_{0}\right) / \mathrm{n}}-\frac{1}{2 \mathrm{n}} \text { or } \\
& \hat{\mathrm{p}}<\mathrm{p}_{0}-\mathrm{z}_{\mathrm{a} / 2} \sqrt{\mathrm{p}_{0}\left(1-\mathrm{p}_{0}\right) / \mathrm{n}}+\frac{1}{2 \mathrm{n}}
\end{aligned}
$$

Method 7-Recentered Wald interval without continuity: We will reject the null hypothesis in (1) at a level of significance when:

$$
\begin{aligned}
& \hat{\mathrm{p}}<\frac{\mathrm{X}_{0}+\mathrm{z}_{\mathrm{a} / 2}^{2}}{\mathrm{n}+\mathrm{z}_{\mathrm{a} / 2}^{2}}-\mathrm{z}_{\mathrm{a} / 2} \sqrt{\frac{\mathrm{X}_{0}}{\mathrm{n}^{2}}\left(1-\frac{\mathrm{X}_{0}}{\mathrm{n}}\right)} \text { or } \\
& \hat{\mathrm{p}}<\frac{\mathrm{X}_{0}+\mathrm{z}_{\mathrm{a} / 2}^{2}}{\mathrm{n}+\mathrm{z}_{\mathrm{a} / 2}^{2}}+\mathrm{z}_{\mathrm{a} / 2} \sqrt{\frac{\mathrm{X}_{0}}{\mathrm{n}^{2}}\left(1-\frac{\mathrm{X}_{0}}{\mathrm{n}}\right)}
\end{aligned}
$$


Table 1: Proposed test statistics $(\mathrm{X} 0=\mathrm{np} 0$ where $0<\mathrm{p} 0<1)$

\begin{tabular}{|c|c|c|}
\hline Method & Lower critical value & Upper critical value \\
\hline 1 & $\operatorname{BetaX}_{0, \mathrm{n}-\mathrm{X} 0+1, \mathrm{a} / 2}$ & $\operatorname{BetaX}_{0+1, \mathrm{n}-\mathrm{X} 0,1-\mathrm{a}=2}$ \\
\hline 2 & $\operatorname{BetaX}_{0+1, \mathrm{n}-\mathrm{X} 0+1, \mathrm{a}=2}$ & $\operatorname{BetaX}_{0+1, \mathrm{n}-\mathrm{X} 0+1,1-\mathrm{a}=2}$ \\
\hline \multirow[t]{2}{*}{3} & $2 \mathrm{x}_{0}+\mathrm{z}_{\mathrm{a} / 2}^{2}-\mathrm{z}_{\mathrm{a} / 2} \sqrt{\mathrm{z}_{\mathrm{a} / 2}^{2}+4 \mathrm{X}_{0}\left(1-\mathrm{x}_{0} / \mathrm{n}\right)}$ & $2 \mathrm{x}_{0}+\mathrm{z}_{\mathrm{a} / 2}^{2}+\mathrm{z}_{\mathrm{a} / 2} \sqrt{\mathrm{z}_{\mathrm{a} / 2}^{2}+4 \mathrm{X}_{0}\left(1-\mathrm{x}_{0} / \mathrm{n}\right)}$ \\
\hline & $2 \quad 2\left(\mathrm{n}+\mathrm{z}_{\mathrm{a} / 2}^{2}\right)$ & $2\left(\mathrm{n}+\mathrm{z}_{\mathrm{a} / 2}^{2}\right)$ \\
\hline \multirow{3}{*}{4} & $2 \mathrm{x}_{0}+\mathrm{z}_{\mathrm{a} / 2}^{2}-1-\mathrm{z}_{\mathrm{a} / 2} \sqrt{\mathrm{a}(\mathrm{x})}$ & $2 \mathrm{X}_{0}+\mathrm{z}_{\mathrm{a} / 2}^{2}+1+\mathrm{z}_{\mathrm{a} / 2} \sqrt{\mathrm{b}(\mathrm{x})}$ \\
\hline & $2\left(\mathrm{n}+\mathrm{z}_{\mathrm{a} / 2}^{2}\right)$ & $2\left(\mathrm{n}+\mathrm{z}_{\mathrm{a} / 2}^{2}\right)$ \\
\hline & $\mathrm{a}(\mathrm{x})=\mathrm{z}_{\mathrm{a} / 2}^{2}-\left(2+\frac{1}{\mathrm{n}}+4 \mathrm{X}_{0}\left(\left(1-\frac{\mathrm{x}_{0}}{\mathrm{n}}+\frac{1}{\mathrm{n}}\right)\right)\right.$ & $\mathrm{b}(\mathrm{x})=\mathrm{z}_{\mathrm{a} / 2}^{2}+\left(2-\frac{1}{\mathrm{n}}+4 \mathrm{X}_{0}\left(\left(1-\frac{\mathrm{X}_{0}}{\mathrm{n}}-\frac{1}{\mathrm{n}}\right)\right)\right.$ \\
\hline 5 & $\frac{\mathrm{x}_{0}}{\mathrm{n}}-\mathrm{z}_{\mathrm{a} / 2} \sqrt{\frac{\mathrm{x}_{0}}{\mathrm{n}^{2}}\left(1-\frac{\mathrm{x}_{0}}{\mathrm{n}}\right)}$ & $\frac{\mathrm{x}_{0}}{\mathrm{n}}+\mathrm{z}_{\mathrm{a} / 2} \sqrt{\frac{\mathrm{x}_{0}}{\mathrm{n}^{2}}\left(1-\frac{\mathrm{x}_{0}}{\mathrm{n}}\right)}$ \\
\hline 6 & $\frac{\mathrm{x}_{0}}{\mathrm{n}}+\mathrm{z}_{\mathrm{a} / 2} \sqrt{\frac{\mathrm{x}_{0}}{\mathrm{n}^{2}}\left(1-\frac{\mathrm{X}_{0}}{\mathrm{n}}\right)-\frac{1}{2 \mathrm{n}}}$ & $\frac{\mathrm{x}_{0}}{\mathrm{n}}+\mathrm{z}_{\mathrm{a} / 2} \sqrt{\frac{\mathrm{x}_{0}}{\mathrm{n}^{2}}\left(1-\frac{\mathrm{x}_{0}}{\mathrm{n}}\right)}+\frac{1}{2 \mathrm{n}}$ \\
\hline 7 & $\left.\frac{x_{0}+z_{a / 2}^{2}}{n+z_{a / 2}^{2}}-z_{a / 2} \sqrt{\frac{x_{0}}{n^{2}}\left(1-\frac{x_{0}}{n}\right.}\right)$ & $\frac{x_{0}+z_{a / 2}^{2}}{n+z_{a / 2}^{2}}+z_{a / 2} \sqrt{\frac{x_{0}}{n^{2}}\left(1-\frac{x_{0}}{n}\right)}$ \\
\hline 8 & $\frac{x_{0}+z_{a / 2}^{2}}{n+z_{a / 2}^{2}}-z_{a / 2} \sqrt{\frac{x_{0}}{n}\left(1-\frac{x_{0}}{n}\right)}-\frac{1}{2 n}$ & $\frac{\mathrm{x}_{0}+\mathrm{z}_{\mathrm{a} / 2}^{2}}{\mathrm{n}+\mathrm{z}_{\mathrm{a} / 2}^{2}}+\mathrm{z}_{\mathrm{a} / 2} \sqrt{\frac{\mathrm{x}_{0}}{\mathrm{n}^{2}}\left(1-\frac{\mathrm{x}_{0}}{\mathrm{n}}\right)}+-\frac{1}{2 \mathrm{n}}$ \\
\hline \multirow{2}{*}{9} & $\operatorname{Bin}_{\mathrm{n}, \mathrm{X} 0 / \mathrm{n}, \mathrm{a} / 2}$ & $\operatorname{Bin}_{\mathrm{n}, \mathrm{X} 0 / \mathrm{n}, 1-\mathrm{a} / 2}$ \\
\hline & $\mathrm{n}$ & $\mathrm{n}$ \\
\hline \multirow{2}{*}{10} & $\operatorname{Bin}_{\mathrm{n}, \mathrm{X} 0 / \mathrm{n}, \mathrm{a} / 2}-1$ & $\operatorname{Bin}_{\mathrm{n}, \mathrm{X} 0 / \mathrm{n}, 1-\mathrm{a} / 2}$ \\
\hline & $\mathrm{n} \quad 2 \mathrm{n}$ & $\mathrm{n} \quad 2 \mathrm{n}$ \\
\hline 11 & $\sin ^{2}\left(\arcsin \sqrt{\frac{x_{0}}{n}}-\frac{z_{a} / 2}{2 \sqrt{n}}\right)$ & $\sin ^{2}\left(\arcsin \sqrt{\left.\frac{x_{0}}{n}+\frac{z_{a / 2}}{2 \sqrt{n}}\right)}\right.$ \\
\hline 12 & $\sin ^{2}\left(\arcsin \sqrt{\left.\frac{\mathrm{X}_{0}+0.5}{\mathrm{n}}-\frac{\mathrm{z}_{\mathrm{a} / 2}}{2 \sqrt{\mathrm{n}}}\right)}\right.$ & $\sin ^{2}\left(\arcsin \sqrt{\frac{X_{0}+0.5}{n}}+\frac{z_{a / 2}}{2 \sqrt{n}}\right)$ \\
\hline \multirow[t]{2}{*}{13} & $\sin ^{2}\left(\arcsin \sqrt{3 / 8+x_{0}-0.5}-\frac{z_{a / 2}}{\square}\right)$ & $\sin ^{2}\left(\arcsin \sqrt{\frac{3 / 8+X_{0}-0.5}{2}+}\right.$ \\
\hline & $\sqrt{n+3 / 4} \quad 2 \sqrt{n+1 / 2}$ & $n+3 / 4$ \\
\hline \multirow[t]{4}{*}{14} & \begin{tabular}{l|l}
$x_{0}+2$ \\
$z_{a} / 2$
\end{tabular} & \\
\hline & $n+4 \quad \sqrt[a]{2} \sqrt{(n+4)^{2}} \quad n+4$ & \\
\hline & $\frac{x_{0}+2}{+z_{a} / 2} \sqrt{\frac{x_{0}+2}{(1-2}\left(1-\frac{x_{0}+2}{2}\right)}$ & \\
\hline & $\mathrm{n}+4 \quad \sqrt{(\mathrm{n}+4)^{2}} \quad \mathrm{n}+4$ & \\
\hline
\end{tabular}

Method 8-recentered wald interval with continuity correction: We will reject the null hypothesis in (1) at a level of significance when:

$$
\begin{aligned}
& \hat{\mathrm{p}}<\frac{\mathrm{X}_{0}+\mathrm{z}_{\mathrm{a} / 2}^{2}}{\mathrm{n}+\mathrm{z}_{\mathrm{a} / 2}^{2}}-\mathrm{z}_{\mathrm{a} / 2} \sqrt{\frac{\mathrm{X}_{0}}{\mathrm{n}^{2}}\left(1-\frac{\mathrm{X}_{0}}{\mathrm{n}}\right)-\frac{1}{2 \mathrm{n}}} \text { or } \\
& \hat{\mathrm{p}}>\frac{\mathrm{X}_{0}+\mathrm{z}_{\mathrm{a} / 2}^{2}}{\mathrm{n}+\mathrm{z}_{\mathrm{a} / 2}^{2}}+\mathrm{z}_{\mathrm{a} / 2} \sqrt{\frac{\mathrm{X}_{0}}{\mathrm{n}^{2}}\left(1-\frac{\mathrm{X}_{0}}{\mathrm{n}}\right)}+\frac{1}{2 \mathrm{n}}
\end{aligned}
$$

Method 9- Bootstrap Method without continuity correction: We will reject the null hypothesis in (1) at a level of significance when:

$$
\hat{\mathrm{p}}<\frac{\operatorname{Bin}_{\mathrm{n}, \mathrm{X} 0 / \mathrm{n}, \mathrm{a} / 2}}{\mathrm{n}} \text { or } \hat{\mathrm{p}}<\frac{\operatorname{Bin}_{\mathrm{n}, \mathrm{X} 0 / \mathrm{n}, 1-\mathrm{a} / 2}}{\mathrm{n}}
$$

Method 10-Bootstrap Method with continuity correction: We will reject the null hypothesis in (1) at a level of significance when:

$\hat{\mathrm{p}}<\frac{\operatorname{Bin}_{\mathrm{n}, \mathrm{X} 0 / \mathrm{n}, \mathrm{a} / 2}}{\mathrm{n}}-\frac{1}{2 \mathrm{n}}$ or $\hat{\mathrm{p}}<\frac{\operatorname{Bin}_{\mathrm{n}, \mathrm{X} 0 / \mathrm{n}, 1-\mathrm{a} / 2}}{\mathrm{n}}+\frac{1}{2 \mathrm{n}}$

Method 11-method based on arcsine (variance stabilizing) transformation: We will reject the null hypothesis in (1) at a level of significance when:

$$
\begin{aligned}
& \sin ^{2}\left(\arcsin \hat{\mathrm{p}}<\sqrt{\frac{\mathrm{X}_{0}}{\mathrm{n}}}-\frac{\mathrm{z}_{\mathrm{a} / 2}}{2 \sqrt{\mathrm{n}}}\right. \text { or } \\
& \sin ^{2}\left(\arcsin \sqrt{\frac{\mathrm{X}_{0}}{\mathrm{n}}}+\frac{\mathrm{z}_{\mathrm{a} / 2}}{2 \sqrt{\mathrm{n}}}\right.
\end{aligned}
$$


Method 12-Method based on arcsine (variance stabilizing) transformation with a continuity correction: We will reject the null hypothesis in (1) at a level of significance when:

$$
\begin{aligned}
& \hat{\mathrm{p}}<\sin ^{2}\left(\arcsin \sqrt{\frac{\mathrm{X}_{0}-0.5}{\mathrm{n}}}-\frac{\mathrm{z}_{\mathrm{a} / 2}}{2 \sqrt{\mathrm{n}}}\right. \text { or } \\
& \hat{\mathrm{p}}>\sin ^{2}\left(\arcsin \sqrt{\frac{\mathrm{X}_{0}+0.5}{\mathrm{n}}}+\frac{\mathrm{z}_{\mathrm{a} / 2}}{2 \sqrt{\mathrm{n}}}\right.
\end{aligned}
$$

Method 13method based on arcsine (variance stabilizing) transformation with another continuity correction: We will reject the null hypothesis in (1) at a level of significance when:

$$
\begin{aligned}
& \hat{\mathrm{p}}<\sin ^{2}\left(\arcsin \sqrt{\frac{3 / 8+\mathrm{X}_{0}-0.5}{\mathrm{n}+3 / 4}}-\frac{\mathrm{z}_{\mathrm{a} / 2}}{2 \sqrt{\mathrm{n}+1 / 2}}\right. \text { or } \\
& \hat{\mathrm{p}}>\sin ^{2}\left(\arcsin \sqrt{\frac{3 / 8+\mathrm{X}_{0}+0.5}{\mathrm{n}+3 / 4}}+\frac{\mathrm{z}_{\mathrm{a} / 2}}{2 \sqrt{\mathrm{n}+1 / 2}}\right.
\end{aligned}
$$

Method 14- Add 4 method: We will reject the null hypothesis in (1) at a level of significance when:

$$
\begin{aligned}
& \hat{\mathrm{p}}<\frac{\mathrm{X}_{0}+2}{\mathrm{n}+4}-\mathrm{z}_{\mathrm{a} / 2} \sqrt{\frac{\mathrm{X}_{0}+2}{(\mathrm{n}+4)^{2}}}\left(1-\frac{\mathrm{X}_{0}+2}{\mathrm{n}+4}\right. \text { or } \\
& \hat{\mathrm{p}}<\frac{\mathrm{X}_{0}+2}{\mathrm{n}+4}-\mathrm{z}_{\mathrm{a} / 2} \sqrt{\frac{\mathrm{X}_{0}+2}{(\mathrm{n}+4)^{2}}}\left(1-\frac{\mathrm{X}_{0}+2}{\mathrm{n}+4}\right.
\end{aligned}
$$
Table 1.

We have summarized all proposed test statistics in

\section{RESULTS}

The main objective of this study is to find some good test statistics for testing the parameter of a binomial distribution. Since a theoretical comparison is not possible, a simulation study has been made to compare the size and power performances of the test statistics.

Simulation technique: The plan of the simulation study is as follows:

- We used sample sizes $n=10,20,30,50$ and 100

- Random samples are generated from a binomial distribution with the following pdf

$$
\begin{aligned}
& \mathrm{p}(\mathrm{x})=\left(\begin{array}{l}
\mathrm{n} \\
\mathrm{x}
\end{array}\right) \mathrm{p}^{\mathrm{z}}(1-\mathrm{p})^{\mathrm{n}-\mathrm{x}} \\
& 0<\mathrm{p}<1, \mathrm{x}=0,1,2 \ldots \mathrm{n}
\end{aligned}
$$

- We generate samples for $p=0,1,0,2,0,3,0,4$, 0,5 .
In each case, 5000 random samples are generated. The most common $5 \%$ level of significance $(\mathrm{a}=0.05)$ is used to compute the empirical power. We compare the performance of the test statistics based on empirical sizes and powers, which is calculated as the fraction of the rejections of the null hypothesis out of 5000 simulation replications. Empirical size and power of the test are calculated based on the following hypothesis, $\mathrm{H}_{\mathrm{a}}: \mathrm{p}_{0} \pm \mathrm{c}$. We get size of the test a when $\mathrm{c}=0$, otherwise powers of the considered test statistics. For $a=0.05$, the simulation results are presented in Table 2-6.

\section{DISCUSSION}

From Table 2-6 we observed a general pattern is that as the sample size increase the power of the test also increase and the nominal size approach to 0.05 . Also power increase as the value of $\mathrm{p}$ departed from the hypothesized value $\mathrm{p}_{0}$. We observed that for large $\mathrm{n}$, the performance of the test statistics do not differ greatly in the sense of power and attaining nominal size of the test. However, a significant difference observed for both small sample size and small p. Overall, based on the empirical power and size of the test, we may conclude that group 1 (Method 6, 8, 10, 12) performed the best followed by group 2(Method 1, 4, 5, 13), group 3 (Method 2, 3, 7, 11 14) and Method 9 performed the worse.

\section{Applications:}

Example 1: Wagenknecht et al. (2003) collected data on a sample of 301 from Hispanic women living in San Antonio, Texas. One variable of interest was the percentage of subjects with Impaired Fasting Glucose (IFG). IFG refers to a metabolic stage intermediate between normal glucose homeostasis and diabetes. In the study 24 women were classified in the IFG stage. The article cites population estimates for IFG among Hispanic women as 6.3 percent. We would like to test whether there is sufficient evidence to indicate that the population of Hispanic women in San Antonio has a prevalence of IFG different from $6.3 \%$. The hypothesis $\mathrm{H}_{0}: \mathrm{p}=0.063$ against $\mathrm{H}_{1}: \mathrm{p} \neq 0.063$. From the sample we have $\hat{p}=24 / 301=0.080$. The results of the test from all the methods discussed here are given in Table 7. All methods leads to the same conclusion of Do not reject $\mathrm{H} 0$, at the significance level 0.05 . However, we might consider those tests have good power and nominal size of the tests.

Example 2: Becker et al. (2003) conducted a study using a sample of 50 ethnic Fijian women. The women completed a self report questionnaire on dieting and attitudes toward body shape and change. 
Am. J. Biostatistics 1 (2): 82-93, 2010

Table 2: Empirical size and power of the test for $\mathrm{n}=10, \mathrm{p}=0.1,0.2$

\begin{tabular}{|c|c|c|c|c|c|c|c|c|c|}
\hline $\begin{array}{l}\text { Method n p0 } \\
n=10, p=0.1\end{array}$ & 0.1 & 0.2 & 0.3 & 0.4 & 0.5 & 0.6 & 0.7 & 0.8 & 0.9 \\
\hline 1 & 0.3550 & 0.3412 & 0.3576 & 0.7376 & 0.7358 & 0.9334 & 0.9864 & 0.9986 & 1.0000 \\
\hline 2 & 0.3550 & 0.3412 & 0.7368 & 0.7376 & 0.9312 & 0.9868 & 0.9864 & 0.9986 & 1.0000 \\
\hline 3 & 0.3550 & 0.3412 & 0.7368 & 0.7376 & 0.9312 & 0.9868 & 0.9864 & 0.9986 & 1.0000 \\
\hline 4 & 0.3550 & 0.3412 & 0.3576 & 0.7376 & 0.9312 & 0.9334 & 0.9864 & 0.9986 & 1.0000 \\
\hline 5 & 0.4154 & 0.3426 & 0.3578 & 0.3622 & 0.7358 & 0.9334 & 0.9990 & 1.0000 & 1.0000 \\
\hline 6 & 0.3632 & 0.3426 & 0.3576 & 0.3622 & 0.7358 & 0.9334 & 0.9864 & 1.0000 & 1.0000 \\
\hline 7 & 0.3632 & 0.3412 & 0.3576 & 0.7376 & 0.7358 & 0.9334 & 0.9864 & 0.9986 & 1.0000 \\
\hline 8 & 0.3550 & 0.3412 & 0.3576 & 0.3622 & 0.7358 & 0.9334 & 0.9864 & 0.9986 & 1.0000 \\
\hline 9 & 0.4154 & 0.3426 & 0.3578 & 0.7376 & 0.9312 & 0.9868 & 0.9990 & 1.0000 & 1.0000 \\
\hline 10 & 0.3632 & 0.3412 & 0.3576 & 0.3622 & 0.7358 & 0.9334 & 0.9864 & 0.9986 & 1.0000 \\
\hline 11 & 0.3632 & 0.3426 & 0.3576 & 0.7376 & 0.9312 & 0.9334 & 0.9864 & 1.0000 & 1.0000 \\
\hline 12 & 0.3550 & 0.3412 & 0.3576 & 0.7376 & 0.7358 & 0.9334 & 0.9864 & 0.9986 & 1.0000 \\
\hline 13 & 0.3550 & 0.3412 & 0.3576 & 0.7376 & 0.7358 & 0.9334 & 0.9864 & 0.9986 & 1.0000 \\
\hline 14 & 0.3550 & 0.3412 & 0.7368 & 0.7376 & 0.9312 & 0.9868 & 0.9864 & 0.9986 & 1.0000 \\
\hline \multicolumn{10}{|l|}{$\mathrm{n}=10, \mathrm{p}=0.2$} \\
\hline 1 & 0.1416 & 0.1192 & 0.1052 & 0.3746 & 0.3736 & 0.6716 & 0.8842 & 0.9646 & 0.9932 \\
\hline 2 & 0.1416 & 0.1192 & 0.3708 & 0.3750 & 0.6814 & 0.8798 & 0.8842 & 0.9646 & 0.9932 \\
\hline 3 & 0.1416 & 0.1192 & 0.3708 & 0.3750 & 0.6814 & 0.8798 & 0.8842 & 0.9646 & 0.9932 \\
\hline 4 & 0.1416 & 0.1192 & 0.1052 & 0.3746 & 0.6814 & 0.6716 & 0.8842 & 0.9646 & 0.9932 \\
\hline 5 & 0.4318 & 0.1462 & 0.1118 & 0.1002 & 0.3736 & 0.6716 & 0.9666 & 0.9932 & 1.0000 \\
\hline 6 & 0.2302 & 0.1462 & 0.1052 & 0.1002 & 0.3736 & 0.6716 & 0.8842 & 0.9932 & 0.9986 \\
\hline 7 & 0.2302 & 0.1192 & 0.1052 & 0.3746 & 0.3736 & 0.6716 & 0.8842 & 0.9646 & 0.9986 \\
\hline 8 & 0.1416 & 0.1192 & 0.1052 & 0.1002 & 0.3736 & 0.6716 & 0.8842 & 0.9646 & 0.9932 \\
\hline 9 & 0.4318 & 0.1462 & 0.1118 & 0.3750 & 0.6814 & 0.8798 & 0.9666 & 0.9932 & 1.0000 \\
\hline 10 & 0.2302 & 0.1192 & 0.1052 & 0.1002 & 0.3736 & 0.6716 & 0.8842 & 0.9646 & 0.9986 \\
\hline 11 & 0.2302 & 0.1462 & 0.1052 & 0.3746 & 0.6814 & 0.6716 & 0.8842 & 0.9932 & 0.9986 \\
\hline 12 & 0.1416 & 0.1192 & 0.1052 & 0.3746 & 0.3736 & 0.6716 & 0.8842 & 0.9646 & 0.9932 \\
\hline 13 & 0.1416 & 0.1192 & 0.1052 & 0.3746 & 0.3736 & 0.6716 & 0.8842 & 0.9646 & 0.9932 \\
\hline 14 & 0.1416 & 0.1192 & 0.3708 & 0.3750 & 0.6814 & 0.8798 & 0.8842 & 0.9646 & 0.9932 \\
\hline \multicolumn{10}{|l|}{$\mathrm{n}=10, \mathrm{p}=0.3$} \\
\hline 1 & 0.1770 & 0.0768 & 0.0396 & 0.1478 & 0.1424 & 0.3902 & 0.6522 & 0.8604 & 0.9546 \\
\hline 2 & 0.1770 & 0.0768 & 0.1676 & 0.1544 & 0.3746 & 0.6518 & 0.6522 & 0.8604 & 0.9546 \\
\hline 3 & 0.1770 & 0.0768 & 0.1676 & 0.1544 & 0.3746 & 0.6518 & 0.6522 & 0.8604 & 0.9546 \\
\hline 4 & 0.1770 & 0.0768 & 0.0396 & 0.1478 & 0.3746 & 0.3902 & 0.6522 & 0.8604 & 0.9546 \\
\hline 5 & 0.6398 & 0.1846 & 0.0722 & 0.0320 & 0.1424 & 0.3900 & 0.8472 & 0.9528 & 0.9978 \\
\hline 6 & 0.3804 & 0.1846 & 0.0396 & 0.0320 & 0.1424 & 0.3900 & 0.6522 & 0.9528 & 0.9902 \\
\hline 7 & 0.3804 & 0.0768 & 0.0396 & 0.1478 & 0.1424 & 0.3902 & 0.6522 & 0.8604 & 0.9902 \\
\hline 8 & 0.1770 & 0.0768 & 0.0396 & 0.0320 & 0.1424 & 0.3900 & 0.6522 & 0.8604 & 0.9546 \\
\hline 9 & 0.6398 & 0.1846 & 0.0722 & 0.1544 & 0.3746 & 0.6518 & 0.8472 & 0.9528 & 0.9978 \\
\hline 10 & 0.3804 & 0.0768 & 0.0396 & 0.0320 & 0.1424 & 0.3900 & 0.6522 & 0.8604 & 0.9902 \\
\hline 11 & 0.3804 & 0.1846 & 0.0396 & 0.1478 & 0.3746 & 0.3902 & 0.6522 & 0.9528 & 0.9902 \\
\hline 12 & 0.1770 & 0.0768 & 0.0396 & 0.1478 & 0.1424 & 0.3902 & 0.6522 & 0.8604 & 0.9546 \\
\hline 13 & 0.1770 & 0.0768 & 0.0396 & 0.1478 & 0.1424 & 0.3902 & 0.6522 & 0.8604 & 0.9546 \\
\hline 14 & 0.1770 & 0.0768 & 0.1676 & 0.1544 & 0.3746 & 0.6518 & 0.6522 & 0.8604 & 0.9546 \\
\hline \multicolumn{10}{|l|}{$\mathrm{n}=10, \mathrm{p}=0.4$} \\
\hline 1 & 0.3724 & 0.1806 & 0.0624 & 0.0562 & 0.0478 & 0.1624 & 0.3712 & 0.6450 & 0.8338 \\
\hline 2 & 0.3724 & 0.1806 & 0.1046 & 0.1040 & 0.1738 & 0.3778 & 0.3736 & 0.6450 & 0.8338 \\
\hline 3 & 0.3724 & 0.1806 & 0.1046 & 0.1040 & 0.1738 & 0.3778 & 0.3736 & 0.6450 & 0.8338 \\
\hline 4 & 0.3724 & 0.1806 & 0.0624 & 0.0562 & 0.1738 & 0.1624 & 0.3712 & 0.6450 & 0.8338 \\
\hline 5 & 0.8328 & 0.3736 & 0.1676 & 0.0182 & 0.0478 & 0.1612 & 0.6196 & 0.8398 & 0.9878 \\
\hline 6 & 0.6276 & 0.3736 & 0.0624 & 0.0182 & 0.0478 & 0.1612 & 0.3712 & 0.8398 & 0.9486 \\
\hline 7 & 0.6276 & 0.1806 & 0.0624 & 0.0562 & 0.0478 & 0.1624 & 0.3712 & 0.6450 & 0.9486 \\
\hline 8 & 0.3724 & 0.1806 & 0.0624 & 0.0182 & 0.0478 & 0.1612 & 0.3712 & 0.6450 & 0.8338 \\
\hline 9 & 0.8328 & 0.3736 & 0.1676 & 0.1040 & 0.1738 & 0.3778 & 0.6196 & 0.8398 & 0.9878 \\
\hline 10 & 0.6276 & 0.1806 & 0.0624 & 0.0182 & 0.0478 & 0.1612 & 0.3712 & 0.6450 & 0.9486 \\
\hline 11 & 0.6276 & 0.3736 & 0.0624 & 0.0562 & 0.1738 & 0.1624 & 0.3712 & 0.8398 & 0.9486 \\
\hline 12 & 0.3724 & 0.1806 & 0.0624 & 0.0562 & 0.0478 & 0.1624 & 0.3712 & 0.6450 & 0.8338 \\
\hline 13 & 0.3724 & 0.1806 & 0.0624 & 0.0562 & 0.0478 & 0.1624 & 0.3712 & 0.6450 & 0.8338 \\
\hline 14 & 0.3724 & 0.1806 & 0.1046 & 0.1040 & 0.1738 & 0.3778 & 0.3736 & 0.6450 & 0.8338 \\
\hline \multicolumn{10}{|l|}{$\mathrm{n}=10, \mathrm{p}=0.5$} \\
\hline 1 & 0.6242 & 0.3830 & 0.1748 & 0.0662 & 0.0240 & 0.0638 & 0.1778 & 0.3642 & 0.6266 \\
\hline 2 & 0.6242 & 0.3830 & 0.1860 & 0.1784 & 0.1084 & 0.1828 & 0.1870 & 0.3642 & 0.6266 \\
\hline 3 & 0.6242 & 0.3830 & 0.1860 & 0.1784 & 0.1084 & 0.1828 & 0.1870 & 0.3642 & 0.6266 \\
\hline
\end{tabular}


Am. J. Biostatistics 1 (2): 82-93, 2010

\begin{tabular}{|c|c|c|c|c|c|c|c|c|c|}
\hline 4 & 0.6242 & 0.3830 & 0.1748 & 0.0662 & 0.1084 & 0.0638 & 0.1778 & 0.3642 & 0.6266 \\
\hline 5 & 0.9436 & 0.6230 & 0.3860 & 0.0566 & 0.0240 & 0.0526 & 0.3856 & 0.6224 & 0.9438 \\
\hline 6 & 0.8290 & 0.6230 & 0.1748 & 0.0566 & 0.0240 & 0.0526 & 0.1778 & 0.6224 & 0.8310 \\
\hline 7 & 0.8290 & 0.3830 & 0.1748 & 0.0662 & 0.0240 & 0.0638 & 0.1778 & 0.3642 & 0.8310 \\
\hline 8 & 0.6242 & 0.3830 & 0.1748 & 0.0566 & 0.0240 & 0.0526 & 0.1778 & 0.3642 & 0.6266 \\
\hline 9 & 0.9436 & 0.6230 & 0.3860 & 0.1784 & 0.1084 & 0.1828 & 0.3856 & 0.6224 & 0.9438 \\
\hline 10 & 0.8290 & 0.3830 & 0.1748 & 0.0566 & 0.0240 & 0.0526 & 0.1778 & 0.3642 & 0.8310 \\
\hline 11 & 0.8290 & 0.6230 & 0.1748 & 0.0662 & 0.1084 & 0.0638 & 0.1778 & 0.6224 & 0.8310 \\
\hline 12 & 0.6242 & 0.3830 & 0.1748 & 0.0662 & 0.0240 & 0.0638 & 0.1778 & 0.3642 & 0.6266 \\
\hline 13 & 0.6242 & 0.3830 & 0.1748 & 0.0662 & 0.0240 & 0.0638 & 0.1778 & 0.3642 & 0.6266 \\
\hline 14 & 0.6242 & 0.3830 & 0.1860 & 0.1784 & 0.1084 & 0.1828 & 0.1870 & 0.3642 & 0.6266 \\
\hline
\end{tabular}

Table 3: Empirical size and power of the test for $n=20, p=0.1,0.2$

\begin{tabular}{|c|c|c|c|c|c|c|c|c|c|}
\hline $\begin{array}{l}\text { Method n p0 } \\
\mathrm{n}=20, \mathrm{p}=0.1\end{array}$ & 0.1 & 0.2 & 0.3 & 0.4 & 0.5 & 0.6 & 0.7 & 0.8 & 0.9 \\
\hline 1 & 0.1318 & 0.3932 & 0.6754 & 0.8626 & 0.9860 & 0.9996 & 1.0000 & 1.0000 & 1.0000 \\
\hline 2 & 0.1318 & 0.3932 & 0.6754 & 0.9524 & 0.9860 & 0.9996 & 1.0000 & 1.0000 & 1.0000 \\
\hline 3 & 0.1318 & 0.3932 & 0.6754 & 0.9524 & 0.9860 & 0.9996 & 1.0000 & 1.0000 & 1.0000 \\
\hline 4 & 0.1318 & 0.3932 & 0.6754 & 0.8626 & 0.9860 & 0.9996 & 1.0000 & 1.0000 & 1.0000 \\
\hline 5 & 0.1728 & 0.1164 & 0.3928 & 0.8626 & 0.9860 & 0.9996 & 1.0000 & 1.0000 & 1.0000 \\
\hline 6 & 0.1394 & 0.1162 & 0.3928 & 0.8626 & 0.9860 & 0.9996 & 1.0000 & 1.0000 & 1.0000 \\
\hline 7 & 0.1394 & 0.3932 & 0.6754 & 0.9524 & 0.9860 & 0.9996 & 1.0000 & 1.0000 & 1.0000 \\
\hline 8 & 0.1318 & 0.1162 & 0.6754 & 0.8626 & 0.9860 & 0.9962 & 0.9998 & 1.0000 & 1.0000 \\
\hline 9 & 0.1728 & 0.3934 & 0.6754 & 0.9524 & 0.9960 & 1.0000 & 1.0000 & 1.0000 & 1.0000 \\
\hline 10 & 0.1394 & 0.1162 & 0.3928 & 0.8626 & 0.9860 & 0.9996 & 1.0000 & 1.0000 & 1.0000 \\
\hline 11 & 0.1394 & 0.3934 & 0.6754 & 0.9524 & 0.9860 & 0.9996 & 1.0000 & 1.0000 & 1.0000 \\
\hline 12 & 0.1318 & 0.1162 & 0.6754 & 0.8626 & 0.9860 & 0.9996 & 1.0000 & 1.0000 & 1.0000 \\
\hline 13 & 0.1318 & 0.3932 & 0.6754 & 0.8626 & 0.9860 & 0.9996 & 1.0000 & 1.0000 & 1.0000 \\
\hline $\begin{array}{l}14 \\
n=20, p=0.2\end{array}$ & 0.1318 & 0.3932 & 0.6754 & 0.9524 & 0.9860 & 0.9996 & 1.0000 & 1.0000 & 1.0000 \\
\hline 1 & 0.0980 & 0.0760 & 0.2022 & 0.4016 & 0.7996 & 0.9698 & 0.9964 & 0.9998 & 1.0000 \\
\hline 2 & 0.0980 & 0.0760 & 0.2022 & 0.6198 & 0.7996 & 0.9698 & 0.9964 & 0.9998 & 1.0000 \\
\hline 3 & 0.0980 & 0.0760 & 0.2022 & 0.6198 & 0.7996 & 0.9698 & 0.9964 & 0.9998 & 1.0000 \\
\hline 4 & 0.0980 & 0.0760 & 0.2022 & 0.4016 & 0.7996 & 0.9698 & 0.9964 & 0.9998 & 1.0000 \\
\hline 5 & 0.3828 & 0.0442 & 0.0692 & 0.4016 & 0.7996 & 0.9698 & 0.9964 & 1.0000 & 1.0000 \\
\hline 6 & 0.2102 & 0.0186 & 0.0692 & 0.4016 & 0.7996 & 0.9698 & 0.9964 & 0.9998 & 1.0000 \\
\hline 7 & 0.2102 & 0.0760 & 0.2022 & 0.6198 & 0.7996 & 0.9698 & 0.9964 & 0.9998 & 1.0000 \\
\hline 8 & 0.0980 & 0.0186 & 0.2020 & 0.4016 & 0.7996 & 0.9108 & 0.9884 & 0.9998 & 1.0000 \\
\hline 9 & 0.3828 & 0.1016 & 0.2054 & 0.6198 & 0.9050 & 0.9902 & 0.9996 & 1.0000 & 1.0000 \\
\hline 10 & 0.2102 & 0.0186 & 0.0692 & 0.4016 & 0.7996 & 0.9698 & 0.9964 & 0.9998 & 1.0000 \\
\hline 11 & 0.2102 & 0.1016 & 0.2022 & 0.6198 & 0.7996 & 0.9698 & 0.9964 & 1.0000 & 1.0000 \\
\hline 12 & 0.0980 & 0.0186 & 0.2022 & 0.4016 & 0.7996 & 0.9698 & 0.9964 & 0.9998 & 1.0000 \\
\hline 13 & 0.0980 & 0.0760 & 0.2022 & 0.4016 & 0.7996 & 0.9698 & 0.9964 & 0.9998 & 1.0000 \\
\hline $\begin{array}{l}14 \\
\mathrm{n}=20, \mathrm{p}=0.3\end{array}$ & 0.0980 & 0.0760 & 0.2022 & 0.6198 & 0.7996 & 0.9698 & 0.9964 & 0.9998 & 1.0000 \\
\hline 1 & 0.4042 & 0.1208 & 0.0532 & 0.1164 & 0.4118 & 0.7724 & 0.9532 & 0.9960 & 0.9998 \\
\hline 2 & 0.4042 & 0.1208 & 0.0532 & 0.2506 & 0.4118 & 0.7724 & 0.9532 & 0.9960 & 0.9998 \\
\hline 3 & 0.4042 & 0.1208 & 0.0532 & 0.2506 & 0.4118 & 0.7724 & 0.9532 & 0.9960 & 0.9998 \\
\hline 4 & 0.4042 & 0.1208 & 0.0532 & 0.1164 & 0.4118 & 0.7724 & 0.9532 & 0.9960 & 0.9998 \\
\hline 5 & 0.7690 & 0.2240 & 0.0258 & 0.1164 & 0.4118 & 0.7724 & 0.9532 & 0.9986 & 1.0000 \\
\hline 6 & 0.5926 & 0.1128 & 0.0258 & 0.1164 & 0.4118 & 0.7724 & 0.9532 & 0.9960 & 1.0000 \\
\hline 7 & 0.5926 & 0.1208 & 0.0532 & 0.2506 & 0.4118 & 0.7724 & 0.9532 & 0.9960 & 1.0000 \\
\hline 8 & 0.4042 & 0.1128 & 0.0406 & 0.1164 & 0.4118 & 0.6038 & 0.8870 & 0.9960 & 0.9998 \\
\hline 9 & 0.7690 & 0.2320 & 0.0864 & 0.2542 & 0.6112 & 0.8860 & 0.9832 & 0.9986 & 1.0000 \\
\hline 10 & 0.5926 & 0.112 & 80.0258 & 0.1164 & 0.4118 & 0.7724 & 0.9532 & 0.9960 & 1.0000 \\
\hline 11 & 0.5926 & 0.2320 & 0.0532 & 0.2506 & 0.4118 & 0.7724 & 0.9532 & 0.9986 & 1.0000 \\
\hline 12 & 0.4042 & 0.1128 & 0.0532 & 0.1164 & 0.4118 & 0.7724 & 0.9532 & 0.9960 & 0.9998 \\
\hline 13 & 0.4042 & 0.1208 & 0.0532 & 0.1164 & 0.4118 & 0.7724 & 0.9532 & 0.9960 & 0.9998 \\
\hline $\begin{array}{l}14 \\
n=20, p=0.4\end{array}$ & 0.4042 & 0.1208 & 0.0532 & 0.2506 & 0.4118 & 0.7724 & 0.9532 & 0.9960 & 0.9998 \\
\hline 1 & 0.7476 & 0.4070 & 0.1258 & 0.0418 & 0.1370 & 0.4190 & 0.7536 & 0.9426 & 0.9932 \\
\hline 2 & 0.7476 & 0.4070 & 0.1258 & 0.0794 & 0.1370 & 0.4192 & 0.7536 & 0.9426 & 0.9932 \\
\hline 3 & 0.7476 & 0.4070 & 0.1258 & 0.0794 & 0.1370 & 0.4192 & 0.7536 & 0.9426 & 0.9932 \\
\hline 4 & 0.7476 & 0.4070 & 0.1258 & 0.0418 & 0.1370 & 0.4190 & 0.7536 & 0.9426 & 0.9932 \\
\hline 5 & 0.9478 & 0.5940 & 0.1226 & 0.0418 & 0.1370 & 0.4190 & 0.7536 & 0.9790 & 0.9996 \\
\hline
\end{tabular}


Am. J. Biostatistics 1 (2): 82-93, 2010

\begin{tabular}{|c|c|c|c|c|c|c|c|c|c|}
\hline 6 & 0.8750 & 0.4066 & 0.1226 & 0.0418 & 0.1370 & 0.4190 & 0.7536 & 0.9426 & 0.9986 \\
\hline 7 & 0.8750 & 0.4070 & 0.1258 & 0.0794 & 0.1370 & 0.4192 & 0.7536 & 0.9426 & 0.9986 \\
\hline 8 & 0.7476 & 0.4066 & 0.0564 & 0.0252 & 0.1370 & 0.2594 & 0.5898 & 0.9426 & 0.9932 \\
\hline 9 & 0.9478 & 0.5944 & 0.2406 & 0.1200 & 0.2710 & 0.6072 & 0.8710 & 0.9790 & 0.9996 \\
\hline 10 & 0.8750 & 0.4066 & 0.1226 & 0.0418 & 0.1370 & 0.4190 & 0.7536 & 0.9426 & 0.9986 \\
\hline 11 & 0.8750 & 0.5944 & 0.1258 & 0.0794 & 0.1370 & 0.4192 & 0.7536 & 0.9790 & 0.9986 \\
\hline 12 & 0.7476 & 0.4066 & 0.1258 & 0.0418 & 0.1370 & 0.4190 & 0.7536 & 0.9426 & 0.9932 \\
\hline 13 & 0.7476 & 0.4070 & 0.1258 & 0.0418 & 0.1370 & 0.4190 & 0.7536 & 0.9426 & 0.9932 \\
\hline & 0.7476 & 0.4070 & 0.1258 & 0.0794 & 0.1370 & 0.4192 & 0.7536 & 0.9426 & 0.9932 \\
\hline 1 & 0.9396 & 0.7378 & 0.4250 & 0.1246 & 0.0388 & 0.1358 & 0.4144 & 0.7468 & 0.9504 \\
\hline 2 & 0.9396 & 0.7378 & 0.4250 & 0.1282 & 0.0388 & 0.1406 & 0.4144 & 0.7468 & 0.9504 \\
\hline 3 & 0.9396 & 0.7378 & 0.4250 & 0.1282 & 0.0388 & 0.1406 & 0.4144 & 0.7468 & 0.9504 \\
\hline 4 & 0.9396 & 0.7378 & 0.4250 & 0.1246 & 0.0388 & 0.1358 & 0.4144 & 0.7468 & 0.9504 \\
\hline 5 & 0.9926 & 0.8614 & 0.4248 & 0.1246 & 0.0388 & 0.1358 & 0.4144 & 0.8614 & 0.9952 \\
\hline 6 & 0.9766 & 0.7378 & 0.4248 & 0.1246 & 0.0388 & 0.1358 & 0.4144 & 0.7468 & 0.9850 \\
\hline 7 & 0.9766 & 0.7378 & 0.4250 & 0.1282 & 0.0388 & 0.1406 & 0.4144 & 0.7468 & 0.9850 \\
\hline 8 & 0.9396 & 0.7378 & 0.2562 & 0.0542 & 0.0388 & 0.0592 & 0.2628 & 0.7468 & 0.9504 \\
\hline 9 & 0.9926 & 0.8614 & 0.6002 & 0.2516 & 0.1132 & 0.2542 & 0.5800 & 0.8614 & 0.9952 \\
\hline 10 & 0.9766 & 0.7378 & 0.4248 & 0.1246 & 0.0388 & 0.1358 & 0.4144 & 0.7468 & 0.9850 \\
\hline 11 & 0.9766 & 0.8614 & 0.4250 & 0.1282 & 0.0388 & 0.1406 & 0.4144 & 0.8614 & 0.9850 \\
\hline 12 & 0.9396 & 0.7378 & 0.4250 & 0.1246 & 0.0388 & 0.1358 & 0.4144 & 0.7468 & 0.9504 \\
\hline 13 & 0.9396 & 0.7378 & 0.4250 & 0.1246 & 0.0388 & 0.1358 & 0.4144 & 0.7468 & 0.9504 \\
\hline 14 & 0.9396 & 0.7378 & 0.4250 & 0.1282 & 0.0388 & 0.1406 & 0.4144 & 0.7468 & 0.9504 \\
\hline
\end{tabular}

Table 4: Empirical size and power of the test for $\mathrm{n}=30, \mathrm{p}=0.1,0.2$

\begin{tabular}{|c|c|c|c|c|c|c|c|c|c|}
\hline $\begin{array}{l}\text { Method n p0 } \\
\mathrm{n}=30, \mathrm{p}=0.1\end{array}$ & 1 & 0.2 & 0.3 & 0.4 & 0.5 & 0.6 & 0.7 & 0.8 & 0.9 \\
\hline 1 & 0.0522 & 0.4198 & 0.8226 & 0.9754 & 1.0000 & 1.0000 & 1.0000 & 1.0000 & 1.0000 \\
\hline 2 & 0.1912 & 0.4198 & 0.9248 & 0.9920 & 1.0000 & 1.0000 & 1.0000 & 1.0000 & 1.0000 \\
\hline 3 & 0.1912 & 0.4198 & 0.8226 & 0.9920 & 1.0000 & 1.0000 & 1.0000 & 1.0000 & 1.0000 \\
\hline 4 & 0.0456 & 0.4198 & 0.8226 & 0.9754 & 1.0000 & 1.0000 & 1.0000 & 1.0000 & 1.0000 \\
\hline 5 & 0.0720 & 0.1798 & 0.8226 & 0.9754 & 1.0000 & 1.0000 & 1.0000 & 1.0000 & 1.0000 \\
\hline 6 & 0.0720 & 0.1798 & 0.6498 & 0.9754 & 1.0000 & 1.0000 & 1.0000 & 1.0000 & 1.0000 \\
\hline 7 & 0.1912 & 0.4198 & 0.8226 & 0.9920 & 1.0000 & 1.0000 & 1.0000 & 1.0000 & 1.0000 \\
\hline 8 & 0.0456 & 0.4198 & 0.8226 & 0.9754 & 1.0000 & 1.0000 & 1.0000 & 1.0000 & 1.0000 \\
\hline 9 & 0.0720 & 0.4198 & 0.8226 & 0.9920 & 1.0000 & 1.0000 & 1.0000 & 1.0000 & 1.0000 \\
\hline 10 & 0.0522 & 0.1798 & 0.6498 & 0.9754 & 1.0000 & 1.0000 & 1.0000 & 1.0000 & 1.0000 \\
\hline 11 & 0.0720 & 0.4198 & 0.8226 & 0.9920 & 1.0000 & 1.0000 & 1.0000 & 1.0000 & 1.0000 \\
\hline 12 & 0.0522 & 0.4198 & 0.8226 & 0.9754 & 1.0000 & 1.0000 & 1.0000 & 1.0000 & 1.0000 \\
\hline 13 & 0.0522 & 0.4198 & 0.8226 & 0.9754 & 1.0000 & 1.0000 & 1.0000 & 1.0000 & 1.0000 \\
\hline 14 & 0.0522 & 0.4198 & 0.8226 & 0.9920 & 1.0000 & 1.0000 & 1.0000 & 1.0000 & 1.0000 \\
\hline \multicolumn{10}{|l|}{$\mathrm{n}=30, \mathrm{p}=0.2$} \\
\hline 1 & 0.2378 & 0.0504 & 0.2542 & 0.6066 & 0.9346 & 0.9962 & 1.0000 & 1.0000 & 1.0000 \\
\hline 2 & 0.2460 & 0.0504 & 0.4320 & 0.7554 & 0.9346 & 0.9962 & 1.0000 & 1.0000 & 1.0000 \\
\hline 3 & 0.2460 & 0.0504 & 0.2542 & 0.7554 & 0.9346 & 0.9962 & 1.0000 & 1.0000 & 1.0000 \\
\hline 4 & 0.1264 & 0.0504 & 0.2542 & 0.6066 & 0.9346 & 0.9962 & 1.0000 & 1.0000 & 1.0000 \\
\hline 5 & 0.3818 & 0.0356 & 0.2552 & 0.6066 & 0.9346 & 0.9962 & 1.0000 & 1.0000 & 1.0000 \\
\hline 6 & 0.3818 & 0.0356 & 0.1212 & 0.6066 & 0.9346 & 0.9962 & 1.0000 & 1.0000 & 1.0000 \\
\hline 7 & 0.2460 & 0.0504 & 0.2542 & 0.7554 & 0.9346 & 0.9962 & 1.0000 & 1.0000 & 1.0000 \\
\hline 8 & 0.1264 & 0.0504 & 0.2542 & 0.6066 & 0.9346 & 0.9908 & 1.0000 & 1.0000 & 1.0000 \\
\hline 9 & 0.3818 & 0.0670 & 0.2552 & 0.7554 & 0.9722 & 0.9994 & 1.0000 & 1.0000 & 1.0000 \\
\hline 10 & 0.2378 & 0.0190 & 0.1212 & 0.6066 & 0.9346 & 0.9962 & 1.0000 & 1.0000 & 1.0000 \\
\hline 11 & 0.3818 & 0.0670 & 0.2542 & 0.7554 & 0.9346 & 0.9962 & 1.0000 & 1.0000 & 1.0000 \\
\hline 12 & 0.2378 & 0.0504 & 0.2542 & 0.6066 & 0.9346 & 0.9962 & 1.0000 & 1.0000 & 1.0000 \\
\hline 13 & 0.2378 & 0.0504 & 0.2542 & 0.6066 & 0.9346 & 0.9962 & 1.0000 & 1.0000 & 1.0000 \\
\hline 14 & 0.2378 & 0.0504 & 0.2542 & 0.7554 & 0.9346 & 0.9962 & 1.0000 & 1.0000 & 1.0000 \\
\hline \multicolumn{10}{|l|}{$\mathrm{n}=30, \mathrm{p}=0.3$} \\
\hline 1 & 0.7196 & 0.1632 & 0.0412 & 0.1672 & 0.5864 & 0.9200 & 0.9934 & 1.0000 & 1.0000 \\
\hline 2 & 0.7200 & 0.1632 & 0.0860 & 0.2898 & 0.5864 & 0.9200 & 0.9934 & 1.0000 & 1.0000 \\
\hline 3 & 0.7200 & 0.1632 & 0.0412 & 0.2898 & 0.5864 & 0.9200 & 0.9934 & 1.0000 & 1.0000 \\
\hline 4 & 0.5774 & 0.1632 & 0.0412 & 0.1672 & 0.5864 & 0.9200 & 0.9934 & 1.0000 & 1.0000 \\
\hline 5 & 0.8460 & 0.2746 & 0.0596 & 0.1672 & 0.5864 & 0.9200 & 0.9974 & 1.0000 & 1.0000 \\
\hline 6 & 0.8460 & 0.2746 & 0.0226 & 0.1672 & 0.5864 & 0.9200 & 0.9934 & 1.0000 & 1.0000 \\
\hline 7 & 0.7200 & 0.1632 & 0.0412 & 0.2898 & 0.5864 & 0.9200 & 0.9934 & 1.0000 & 1.0000 \\
\hline
\end{tabular}


Am. J. Biostatistics 1 (2): 82-93, 2010

\begin{tabular}{|c|c|c|c|c|c|c|c|c|c|}
\hline 8 & 0.5774 & 0.1632 & 0.0314 & 0.1666 & 0.5864 & 0.8422 & 0.9848 & 1.0000 & 1.0000 \\
\hline 9 & 0.8460 & 0.2768 & 0.0596 & 0.2918 & 0.7252 & 0.9630 & 0.9974 & 1.0000 & 1.0000 \\
\hline 10 & 0.7196 & 0.1610 & 0.0226 & 0.1672 & 0.5864 & 0.9200 & 0.9934 & 1.0000 & 1.0000 \\
\hline 11 & 0.8460 & 0.2768 & 0.0412 & 0.2898 & 0.5864 & 0.9200 & 0.9934 & 1.0000 & 1.0000 \\
\hline 12 & 0.7196 & 0.1632 & 0.0412 & 0.1672 & 0.5864 & 0.9200 & 0.9934 & 1.0000 & 1.0000 \\
\hline 13 & 0.7196 & 0.1632 & 0.0412 & 0.1672 & 0.5864 & 0.9200 & 0.9934 & 1.0000 & 1.0000 \\
\hline \multicolumn{9}{|c|}{$\mathrm{n}=30, \mathrm{p}=0.4$} & 1.0000 \\
\hline 1 & 0.9600 & 0.5680 & 0.1712 & 0.0454 & 0.1718 & 0.5774 & 0.9086 & 0.9930 & 1.0000 \\
\hline 2 & 0.9600 & 0.5680 & 0.1766 & 0.0722 & 0.1718 & 0.5774 & 0.9086 & 0.9930 & 1.0000 \\
\hline 3 & 0.9600 & 0.5680 & 0.1712 & 0.0722 & 0.1718 & 0.5774 & 0.9086 & 0.9930 & 1.0000 \\
\hline 4 & 0.9126 & 0.5680 & 0.1712 & 0.0454 & 0.1718 & 0.5774 & 0.9086 & 0.9930 & 1.0000 \\
\hline 5 & 0.9836 & 0.7030 & 0.2852 & 0.0454 & 0.1718 & 0.5774 & 0.9574 & 0.9974 & 1.0000 \\
\hline 6 & 0.9836 & 0.7030 & 0.1690 & 0.0454 & 0.1718 & 0.5774 & 0.9086 & 0.9974 & 1.0000 \\
\hline 7 & 0.9600 & 0.5680 & 0.1712 & 0.0722 & 0.1718 & 0.5774 & 0.9086 & 0.9930 & 1.0000 \\
\hline 8 & 0.9126 & 0.5680 & 0.0956 & 0.0300 & 0.1718 & 0.4318 & 0.8326 & 0.9930 & 1.0000 \\
\hline 9 & 0.9836 & 0.7030 & 0.2852 & 0.0938 & 0.2896 & 0.7088 & 0.9574 & 0.9974 & 1.0000 \\
\hline 10 & 0.9600 & 0.5680 & 0.1690 & 0.0454 & 0.1718 & 0.5774 & 0.9086 & 0.9930 & 1.0000 \\
\hline 11 & 0.9836 & 0.7030 & 0.1712 & 0.0722 & 0.1718 & 0.5774 & 0.9086 & 0.9974 & 1.0000 \\
\hline 12 & 0.9600 & 0.5680 & 0.1712 & 0.0454 & 0.1718 & 0.5774 & 0.9086 & 0.9930 & 1.0000 \\
\hline 13 & 0.9600 & 0.5680 & 0.1712 & 0.0454 & 0.1718 & 0.5774 & 0.9086 & 0.9930 & 1.0000 \\
\hline 14 & 0.9600 & 0.5680 & 0.1712 & 0.0722 & 0.1718 & 0.5774 & 0.9086 & 0.9930 & 1.0000 \\
\hline \multicolumn{10}{|c|}{$\mathrm{n}=30, \mathrm{p}=0.5$} \\
\hline 1 & 0.9980 & 0.8982 & 0.5742 & 0.1860 & 0.0400 & 0.1728 & 0.5590 & 0.9008 & 0.9960 \\
\hline 2 & 0.9980 & 0.8982 & 0.5744 & 0.1876 & 0.0400 & 0.1754 & 0.5590 & 0.9008 & 0.9960 \\
\hline 3 & 0.9980 & 0.8982 & 0.5742 & 0.1876 & 0.0400 & 0.1754 & 0.5590 & 0.9008 & 0.9960 \\
\hline 4 & 0.9922 & 0.8982 & 0.5742 & 0.1860 & 0.0400 & 0.1728 & 0.5590 & 0.9008 & 0.9906 \\
\hline 5 & 0.9994 & 0.9484 & 0.7094 & 0.1860 & 0.0400 & 0.1728 & 0.6944 & 0.9492 & 0.9984 \\
\hline 6 & 0.9994 & 0.9484 & 0.5742 & 0.1860 & 0.0400 & 0.1728 & 0.5590 & 0.9492 & 0.9984 \\
\hline 7 & 0.9980 & 0.8982 & 0.5742 & 0.1876 & 0.0400 & 0.1754 & 0.5590 & 0.9008 & 0.9960 \\
\hline 8 & 0.9922 & 0.8982 & 0.4346 & 0.1030 & 0.0400 & 0.0960 & 0.4172 & 0.9008 & 0.9906 \\
\hline 9 & 0.9994 & 0.9484 & 0.7094 & 0.2936 & 0.0956 & 0.2840 & 0.6944 & 0.9492 & 0.9984 \\
\hline 10 & 0.9980 & 0.8982 & 0.5742 & 0.1860 & 0.0400 & 0.1728 & 0.5590 & 0.9008 & 0.9960 \\
\hline 11 & 0.9994 & 0.9484 & 0.5742 & 0.1876 & 0.0400 & 0.1754 & 0.5590 & 0.9492 & 0.9984 \\
\hline 12 & 0.9980 & 0.8982 & 0.5742 & 0.1860 & 0.0400 & 0.1728 & 0.5590 & 0.9008 & 0.9960 \\
\hline 13 & 0.9980 & 0.8982 & 0.5742 & 0.1860 & 0.0400 & 0.1728 & 0.5590 & 0.9008 & 0.9960 \\
\hline 14 & 0.9980 & 0.8982 & 0.5742 & 0.1876 & 0.0400 & 0.1754 & 0.5590 & 0.9008 & 0.9960 \\
\hline
\end{tabular}

Table 5: Empirical size and power of the test for $\mathrm{n}=50, \mathrm{p}=0.10,0.20$

\begin{tabular}{|c|c|c|c|c|c|c|c|c|c|}
\hline $\begin{array}{l}\text { Method } \mathrm{n} \text { p0 } \\
\mathrm{n}=50, \mathrm{p}=0.1\end{array}$ & 0.1 & 0.2 & 0.3 & 0.4 & 0.5 & 0.6 & 0.7 & 0.8 & 0.9 \\
\hline 1 & 0.0432 & 0.6208 & 0.9354 & 0.9994 & 0.9998 & 1.0000 & 1.0000 & 1.0000 & 1.0000 \\
\hline 2 & 0.1134 & 0.6208 & 0.9724 & 0.9994 & 1.0000 & 1.0000 & 1.0000 & 1.0000 & 1.0000 \\
\hline 3 & 0.1134 & 0.6208 & 0.9724 & 0.9994 & 1.0000 & 1.0000 & 1.0000 & 1.0000 & 1.0000 \\
\hline 4 & 0.0366 & 0.6208 & 0.9724 & 0.9994 & 0.9998 & 1.0000 & 1.0000 & 1.0000 & 1.0000 \\
\hline 5 & 0.0276 & 0.4398 & 0.9354 & 0.9994 & 1.0000 & 1.0000 & 1.0000 & 1.0000 & 1.0000 \\
\hline 6 & 0.0276 & 0.2608 & 0.9354 & 0.9988 & 0.9998 & 1.0000 & 1.0000 & 1.0000 & 1.0000 \\
\hline 7 & 0.1134 & 0.6208 & 0.9724 & 0.9994 & 1.0000 & 1.0000 & 1.0000 & 1.0000 & 1.0000 \\
\hline 8 & 0.0366 & 0.6208 & 0.9354 & 0.9994 & 0.9998 & 1.0000 & 1.0000 & 1.0000 & 1.0000 \\
\hline 9 & 0.0908 & 0.6208 & 0.9724 & 0.9994 & 1.0000 & 1.0000 & 1.0000 & 1.0000 & 1.0000 \\
\hline 10 & 0.0276 & 0.4398 & 0.9354 & 0.9988 & 0.9998 & 1.0000 & 1.0000 & 1.0000 & 1.0000 \\
\hline 11 & 0.0556 & 0.6208 & 0.9724 & 0.9994 & 1.0000 & 1.0000 & 1.0000 & 1.0000 & 1.0000 \\
\hline 12 & 0.0432 & 0.4398 & 0.9354 & 0.9994 & 0.9998 & 1.0000 & 1.0000 & 1.0000 & 1.0000 \\
\hline 13 & 0.0432 & 0.4398 & 0.9354 & 0.9994 & 0.9998 & 1.0000 & 1.0000 & 1.0000 & 1.0000 \\
\hline $\begin{array}{l}14 \\
\mathrm{n}=50, \mathrm{p}=0.2\end{array}$ & 0.1134 & 0.6208 & 0.9724 & 0.9994 & 1.0000 & 1.0000 & 1.0000 & 1.0000 & 1.0000 \\
\hline 1 & 0.4246 & 0.0650 & 0.3196 & 0.8910 & 0.9946 & 1.0000 & 1.0000 & 1.0000 & 1.0000 \\
\hline 2 & 0.4270 & 0.0650 & 0.4490 & 0.8910 & 0.9976 & 1.0000 & 1.0000 & 1.0000 & 1.0000 \\
\hline 3 & 0.4270 & 0.0650 & 0.4490 & 0.8910 & 0.9976 & 1.0000 & 1.0000 & 1.0000 & 1.0000 \\
\hline 4 & 0.2946 & 0.0562 & 0.4490 & 0.8910 & 0.9946 & 1.0000 & 1.0000 & 1.0000 & 1.0000 \\
\hline 5 & 0.5658 & 0.0514 & 0.3196 & 0.8910 & 0.9976 & 1.0000 & 1.0000 & 1.0000 & 1.0000 \\
\hline 6 & 0.5658 & 0.0180 & 0.3196 & 0.8176 & 0.9946 & 1.0000 & 1.0000 & 1.0000 & 1.0000 \\
\hline 7 & 0.4270 & 0.0650 & 0.4490 & 0.8910 & 0.9976 & 1.0000 & 1.0000 & 1.0000 & 1.0000 \\
\hline 8 & 0.2946 & 0.0562 & 0.3196 & 0.8910 & 0.9946 & 1.0000 & 1.0000 & 1.0000 & 1.0000 \\
\hline
\end{tabular}


Am. J. Biostatistics 1 (2): 82-93, 2010

\begin{tabular}{|c|c|c|c|c|c|c|c|c|c|}
\hline 9 & 0.7016 & 0.0834 & 0.4490 & 0.8910 & 0.9976 & 1.0000 & 1.0000 & 1.0000 & 1.0000 \\
\hline 10 & 0.5658 & 0.0330 & 0.3196 & 0.8176 & 0.9946 & 1.0000 & 1.0000 & 1.0000 & 1.0000 \\
\hline 11 & 0.5660 & 0.0650 & 0.4490 & 0.8910 & 0.9976 & 1.0000 & 1.0000 & 1.0000 & 1.0000 \\
\hline 12 & 0.4246 & 0.0330 & 0.3196 & 0.8910 & 0.9946 & 1.0000 & 1.0000 & 1.0000 & 1.0000 \\
\hline 13 & 0.4246 & 0.0330 & 0.3196 & 0.8910 & 0.9946 & 1.0000 & 1.0000 & 1.0000 & 1.0000 \\
\hline $\begin{array}{l}14 \\
\mathrm{n}=5\end{array}$ & 0.4270 & 0.0650 & 0.4490 & 0.8910 & 0.9976 & 1.0000 & 1.0000 & 1.0000 & 1.0000 \\
\hline 1 & 0.9194 & 0.3030 & 0.0282 & 0.3322 & 0.7762 & 0.9886 & 1.0000 & 1.0000 & 1.0000 \\
\hline 2 & 0.9194 & 0.3030 & 0.0626 & 0.3324 & 0.8556 & 0.9962 & 1.0000 & 1.0000 & 1.0000 \\
\hline 3 & 0.9194 & 0.3030 & 0.0626 & 0.3324 & 0.8556 & 0.9962 & 1.0000 & 1.0000 & 1.0000 \\
\hline 4 & 0.8622 & 0.2088 & 0.0498 & 0.3322 & 0.7762 & 0.9886 & 1.0000 & 1.0000 & 1.0000 \\
\hline 5 & 0.9584 & 0.4304 & 0.0410 & 0.3324 & 0.8556 & 0.9962 & 1.0000 & 1.0000 & 1.0000 \\
\hline 6 & 0.9584 & 0.3026 & 0.0410 & 0.2254 & 0.7762 & 0.9886 & 1.0000 & 1.0000 & 1.0000 \\
\hline 7 & 0.9194 & 0.3030 & 0.0498 & 0.3322 & 0.8556 & 0.9886 & 1.0000 & 1.0000 & 1.0000 \\
\hline 8 & 0.8622 & 0.2088 & 0.0282 & 0.3322 & 0.7762 & 0.9886 & 1.0000 & 1.0000 & 1.0000 \\
\hline 9 & 0.9830 & 0.4306 & 0.0626 & 0.3324 & 0.8556 & 0.9962 & 1.0000 & 1.0000 & 1.0000 \\
\hline 10 & 0.9584 & 0.3028 & 0.0282 & 0.2254 & 0.7762 & 0.9886 & 1.0000 & 1.0000 & 1.0000 \\
\hline 11 & 0.9584 & 0.3030 & 0.0626 & 0.3324 & 0.8556 & 0.9962 & 1.0000 & 1.0000 & 1.0000 \\
\hline 12 & 0.9194 & 0.3028 & 0.0282 & 0.3322 & 0.7762 & 0.9886 & 1.0000 & 1.0000 & 1.0000 \\
\hline 13 & 0.9194 & 0.3028 & 0.0282 & 0.3322 & 0.7762 & 0.9886 & 1.0000 & 1.0000 & 1.0000 \\
\hline & 0.9194 & 0.3030 & 0.0626 & 0.3324 & 0.8556 & 0.9962 & 1.0000 & 1.0000 & 1.0000 \\
\hline 1 & 0.9970 & 0.8496 & 0.2358 & 0.0452 & 0.2372 & 0.7696 & 0.9846 & 1.0000 & 1.0000 \\
\hline 2 & 0.9970 & 0.8496 & 0.3310 & 0.0612 & 0.3436 & 0.8484 & 0.9936 & 1.0000 & 1.0000 \\
\hline 3 & 0.9970 & 0.8496 & 0.3310 & 0.0612 & 0.3436 & 0.8484 & 0.9936 & 1.0000 & 1.0000 \\
\hline 4 & 0.9928 & 0.7756 & 0.2362 & 0.0452 & 0.2372 & 0.7696 & 0.9846 & 1.0000 & 1.0000 \\
\hline 5 & 0.9986 & 0.9074 & 0.3306 & 0.0612 & 0.3436 & 0.8484 & 0.9936 & 1.0000 & 1.0000 \\
\hline 6 & 0.9986 & 0.8496 & 0.3306 & 0.0318 & 0.2372 & 0.7696 & 0.9936 & 1.0000 & 1.0000 \\
\hline 7 & 0.9970 & 0.8496 & 0.2362 & 0.0452 & 0.3436 & 0.7696 & 0.9846 & 1.0000 & 1.0000 \\
\hline 8 & 0.9928 & 0.7756 & 0.2358 & 0.0452 & 0.2372 & 0.7696 & 0.9846 & 1.0000 & 1.0000 \\
\hline 9 & 0.9998 & 0.9074 & 0.3310 & 0.0612 & 0.3436 & 0.8484 & 0.9936 & 1.0000 & 1.0000 \\
\hline 10 & 0.9986 & 0.8496 & 0.2358 & 0.0318 & 0.2372 & 0.7696 & 0.9846 & 1.0000 & 1.0000 \\
\hline 11 & 0.9986 & 0.8496 & 0.3310 & 0.0612 & 0.3436 & 0.8484 & 0.9936 & 1.0000 & 1.0000 \\
\hline 12 & 0.9970 & 0.8496 & 0.2358 & 0.0452 & 0.2372 & 0.7696 & 0.9846 & 1.0000 & 1.0000 \\
\hline 13 & 0.9970 & 0.8496 & 0.2358 & 0.0452 & 0.2372 & 0.7696 & 0.9846 & 1.0000 & 1.0000 \\
\hline $\begin{array}{l}14 \\
\mathrm{n}=5\end{array}$ & 0.9970 & 0.8496 & 0.3310 & 0.0612 & 0.3436 & 0.8484 & 0.9936 & 1.0000 & 1.0000 \\
\hline 1 & 1.0000 & 0.9926 & 0.7646 & 0.2472 & 0.0308 & 0.2432 & 0.7578 & 0.9922 & 1.0000 \\
\hline 2 & 1.0000 & 0.9926 & 0.8438 & 0.3430 & 0.0570 & 0.3372 & 0.8394 & 0.9922 & 1.0000 \\
\hline 3 & 1.0000 & 0.9926 & 0.8438 & 0.3430 & 0.0570 & 0.3372 & 0.8394 & 0.9922 & 1.0000 \\
\hline 4 & 1.0000 & 0.9836 & 0.7646 & 0.2472 & 0.0308 & 0.2432 & 0.7578 & 0.9860 & 1.0000 \\
\hline 5 & 1.0000 & 0.9970 & 0.8438 & 0.3430 & 0.0570 & 0.3372 & 0.8394 & 0.9970 & 1.0000 \\
\hline 6 & 1.0000 & 0.9926 & 0.8438 & 0.2466 & 0.0308 & 0.2430 & 0.8394 & 0.9922 & 1.0000 \\
\hline 7 & 1.0000 & 0.9926 & 0.7646 & 0.2472 & 0.0570 & 0.2432 & 0.7578 & 0.9922 & 1.0000 \\
\hline 8 & 1.0000 & 0.9836 & 0.7646 & 0.2472 & 0.0308 & 0.2432 & 0.7578 & 0.9860 & 1.0000 \\
\hline 9 & 1.0000 & 0.9970 & 0.8438 & 0.3430 & 0.0570 & 0.3372 & 0.8394 & 0.9970 & 1.0000 \\
\hline 10 & 1.0000 & 0.9926 & 0.7646 & 0.2466 & 0.0308 & 0.2430 & 0.7578 & 0.9922 & 1.0000 \\
\hline 11 & 1.0000 & 0.9926 & 0.8438 & 0.3430 & 0.0570 & 0.3372 & 0.8394 & 0.9922 & 1.0000 \\
\hline 12 & 1.0000 & 0.9926 & 0.7646 & 0.2472 & 0.0308 & 0.2432 & 0.7578 & 0.9922 & 1.0000 \\
\hline 13 & 1.0000 & 0.9926 & 0.7646 & 0.2472 & 0.0308 & 0.2432 & 0.7578 & 0.9922 & 1.0000 \\
\hline 14 & 1.0000 & 0.9926 & 0.8438 & 0.3430 & 0.0570 & 0.3372 & 0.8394 & 0.9922 & 1.0000 \\
\hline
\end{tabular}

Table 6: Empirical size and power of the test for $n=100, p=0.1,0.2$

\begin{tabular}{|c|c|c|c|c|c|c|c|c|c|}
\hline $\begin{array}{l}\text { Method n p0 } \\
\mathrm{n}=100, \mathrm{p}=0.1\end{array}$ & 0.1 & 0.2 & 0.3 & 0.4 & 0.5 & 0.6 & 0.7 & 0.8 & 0.9 \\
\hline 1 & 0.0310 & 0.8152 & 0.9994 & 1.0000 & 1.0000 & 1.0000 & 1.0000 & 1.0000 & 1.0000 \\
\hline 2 & 0.0620 & 0.8898 & 0.9994 & 1.0000 & 1.0000 & 1.0000 & 1.0000 & 1.0000 & 1.0000 \\
\hline 3 & 0.0620 & 0.8898 & 0.9994 & 1.0000 & 1.0000 & 1.0000 & 1.0000 & 1.0000 & 1.0000 \\
\hline 4 & 0.0550 & 0.8152 & 0.9994 & 1.0000 & 1.0000 & 1.0000 & 1.0000 & 1.0000 & 1.0000 \\
\hline 5 & 0.0644 & 0.8152 & 0.9994 & 1.0000 & 1.0000 & 1.0000 & 1.0000 & 1.0000 & 1.0000 \\
\hline 6 & 0.0294 & 0.7146 & 0.9988 & 1.0000 & 1.0000 & 1.0000 & 1.0000 & 1.0000 & 1.0000 \\
\hline 7 & 0.0620 & 0.8898 & 0.9994 & 1.0000 & 1.0000 & 1.0000 & 1.0000 & 1.0000 & 1.0000 \\
\hline 8 & 0.0620 & 0.8152 & 0.9994 & 1.0000 & 1.0000 & 1.0000 & 1.0000 & 1.0000 & 1.0000 \\
\hline 9 & 0.0954 & 0.8152 & 0.9994 & 1.0000 & 1.0000 & 1.0000 & 1.0000 & 1.0000 & 1.0000 \\
\hline
\end{tabular}


Am. J. Biostatistics 1 (2): 82-93, 2010

Table 6: Continue.... for $\mathrm{n}=100, \mathrm{p}=0.1$

\begin{tabular}{|c|c|c|c|c|c|c|c|c|c|}
\hline 10 & 0.0422 & 0.7146 & 0.9988 & 1.0000 & 1.0000 & 1.0000 & 1.0000 & 1.0000 & 1.0000 \\
\hline 11 & 0.0422 & 0.8152 & 0.9994 & 1.0000 & 1.0000 & 1.0000 & 1.0000 & 1.0000 & 1.0000 \\
\hline 12 & 0.0310 & 0.8152 & 0.9994 & 1.0000 & 1.0000 & 1.0000 & 1.0000 & 1.0000 & 1.0000 \\
\hline 13 & 0.0310 & 0.8152 & 0.9994 & 1.0000 & 1.0000 & 1.0000 & 1.0000 & 1.0000 & 1.0000 \\
\hline $\begin{array}{l}14 \\
n=100, p=0.2\end{array}$ & 0.0620 & 0.8898 & 0.9994 & 1.0000 & 1.0000 & 1.0000 & 1.0000 & 1.0000 & 1.0000 \\
\hline 1 & 0.7182 & 0.0360 & 0.6512 & 0.9936 & 1.0000 & 1.0000 & 1.0000 & 1.0000 & 1.0000 \\
\hline 2 & 0.7182 & 0.0640 & 0.6512 & 0.9936 & 1.0000 & 1.0000 & 1.0000 & 1.0000 & 1.0000 \\
\hline 3 & 0.7182 & 0.0640 & 0.6512 & 0.9936 & 1.0000 & 1.0000 & 1.0000 & 1.0000 & 1.0000 \\
\hline 4 & 0.6262 & 0.0360 & 0.6512 & 0.9936 & 1.0000 & 1.0000 & 1.0000 & 1.0000 & 1.0000 \\
\hline 5 & 0.8696 & 0.0580 & 0.6512 & 0.9936 & 1.0000 & 1.0000 & 1.0000 & 1.0000 & 1.0000 \\
\hline 6 & 0.7972 & 0.0318 & 0.5602 & 0.9884 & 1.0000 & 1.0000 & 1.0000 & 1.0000 & 1.0000 \\
\hline 7 & 0.7182 & 0.0640 & 0.6512 & 0.9936 & 1.0000 & 1.0000 & 1.0000 & 1.0000 & 1.0000 \\
\hline 8 & 0.7182 & 0.0360 & 0.6512 & 0.9936 & 1.0000 & 1.0000 & 1.0000 & 1.0000 & 1.0000 \\
\hline 9 & 0.8696 & 0.0580 & 0.6512 & 0.9964 & 1.0000 & 1.0000 & 1.0000 & 1.0000 & 1.0000 \\
\hline 10 & 0.7972 & 0.0318 & 0.5602 & 0.9936 & 1.0000 & 1.0000 & 1.0000 & 1.0000 & 1.0000 \\
\hline 11 & 0.7972 & 0.0428 & 0.6512 & 0.9936 & 1.0000 & 1.0000 & 1.0000 & 1.0000 & 1.0000 \\
\hline 12 & 0.7182 & 0.0428 & 0.6512 & 0.9936 & 1.0000 & 1.0000 & 1.0000 & 1.0000 & 1.0000 \\
\hline 13 & 0.7182 & 0.0360 & 0.6512 & 0.9936 & 1.0000 & 1.0000 & 1.0000 & 1.0000 & 1.0000 \\
\hline $\begin{array}{l}14 \\
n=100, p=0.3\end{array}$ & 0.7182 & 0.0572 & 0.6512 & 0.9936 & 1.0000 & 1.0000 & 1.0000 & 1.0000 & 1.0000 \\
\hline 1 & 0.9978 & 0.5422 & 0.0480 & 0.5560 & 0.9808 & 1.0000 & 1.0000 & 1.0000 & 1.0000 \\
\hline 2 & 0.9978 & 0.6238 & 0.0480 & 0.5560 & 0.9876 & 1.0000 & 1.0000 & 1.0000 & 1.0000 \\
\hline 3 & 0.9978 & 0.6238 & 0.0480 & 0.5560 & 0.9876 & 1.0000 & 1.0000 & 1.0000 & 1.0000 \\
\hline 4 & 0.9942 & 0.5422 & 0.0424 & 0.5560 & 0.9808 & 1.0000 & 1.0000 & 1.0000 & 1.0000 \\
\hline 5 & 0.9998 & 0.7054 & 0.0608 & 0.5560 & 0.9876 & 1.0000 & 1.0000 & 1.0000 & 1.0000 \\
\hline 6 & 0.9986 & 0.6238 & 0.0358 & 0.4672 & 0.9808 & 1.0000 & 1.0000 & 1.0000 & 1.0000 \\
\hline 7 & 0.9978 & 0.6238 & 0.0480 & 0.5560 & 0.9876 & 1.0000 & 1.0000 & 1.0000 & 1.0000 \\
\hline 8 & 0.9978 & 0.5422 & 0.0424 & 0.5560 & 0.9808 & 1.0000 & 1.0000 & 1.0000 & 1.0000 \\
\hline 9 & 0.9998 & 0.7054 & 0.0608 & 0.6422 & 0.9876 & 1.0000 & 1.0000 & 1.0000 & 1.0000 \\
\hline 10 & 0.9986 & 0.6238 & 0.0358 & 0.5560 & 0.9808 & 1.0000 & 1.0000 & 1.0000 & 1.0000 \\
\hline 11 & 0.9986 & 0.6238 & 0.0480 & 0.5560 & 0.9876 & 1.0000 & 1.0000 & 1.0000 & 1.0000 \\
\hline 12 & 0.9978 & 0.6238 & 0.0480 & 0.5560 & 0.9808 & 1.0000 & 1.0000 & 1.0000 & 1.0000 \\
\hline 13 & 0.9978 & 0.5422 & 0.0480 & 0.5560 & 0.9808 & 1.0000 & 1.0000 & 1.0000 & 1.0000 \\
\hline $\begin{array}{l}14 \\
n=100, p=0.4\end{array}$ & 0.9978 & 0.5422 & 0.0480 & 0.5560 & 0.9876 & 1.0000 & 1.0000 & 1.0000 & 1.0000 \\
\hline 1 & 1.0000 & 0.9840 & 0.5306 & 0.0408 & 0.4670 & 0.9696 & 1.0000 & 1.0000 & 1.0000 \\
\hline 2 & 1.0000 & 0.9912 & 0.5306 & 0.0514 & 0.5468 & 0.9808 & 1.0000 & 1.0000 & 1.0000 \\
\hline 3 & 1.0000 & 0.9912 & 0.5306 & 0.0514 & 0.5468 & 0.9808 & 1.0000 & 1.0000 & 1.0000 \\
\hline 4 & 1.0000 & 0.9840 & 0.4530 & 0.0408 & 0.4670 & 0.9696 & 1.0000 & 1.0000 & 1.0000 \\
\hline 5 & 1.0000 & 0.9956 & 0.6112 & 0.0514 & 0.5468 & 0.9808 & 1.0000 & 1.0000 & 1.0000 \\
\hline 6 & 1.0000 & 0.9912 & 0.5304 & 0.0318 & 0.4670 & 0.9696 & 1.0000 & 1.0000 & 1.0000 \\
\hline 7 & 1.0000 & 0.9912 & 0.5306 & 0.0514 & 0.5468 & 0.9808 & 1.0000 & 1.0000 & 1.0000 \\
\hline 8 & 1.0000 & 0.9840 & 0.4530 & 0.0408 & 0.4670 & 0.9696 & 1.0000 & 1.0000 & 1.0000 \\
\hline 9 & 1.0000 & 0.9956 & 0.6112 & 0.0672 & 0.5468 & 0.9808 & 1.0000 & 1.0000 & 1.0000 \\
\hline 10 & 1.0000 & 0.9912 & 0.5304 & 0.0408 & 0.4670 & 0.9696 & 1.0000 & 1.0000 & 1.0000 \\
\hline 11 & 1.0000 & 0.9912 & 0.5306 & 0.0514 & 0.5468 & 0.9808 & 1.0000 & 1.0000 & 1.0000 \\
\hline 12 & 1.0000 & 0.9912 & 0.5306 & 0.0408 & 0.4670 & 0.9696 & 1.0000 & 1.0000 & 1.0000 \\
\hline 13 & 1.0000 & 0.9840 & 0.5306 & 0.0408 & 0.4670 & 0.9696 & 1.0000 & 1.0000 & 1.0000 \\
\hline $\begin{array}{l}14 \\
n=100, p=0.5\end{array}$ & 1.0000 & 0.9840 & 0.5306 & 0.0514 & 0.5468 & 0.9808 & 1.0000 & 1.0000 & 1.0000 \\
\hline 1 & 1.0000 & 1.0000 & 0.9820 & 0.4582 & 0.0340 & 0.4738 & 0.9826 & 1.0000 & 1.0000 \\
\hline 2 & 1.0000 & 1.0000 & 0.9820 & 0.5418 & 0.0530 & 0.5494 & 0.9826 & 1.0000 & 1.0000 \\
\hline 3 & 1.0000 & 1.0000 & 0.9820 & 0.5418 & 0.0530 & 0.5494 & 0.9826 & 1.0000 & 1.0000 \\
\hline 4 & 1.0000 & 1.0000 & 0.9722 & 0.4582 & 0.0340 & 0.4738 & 0.9720 & 1.0000 & 1.0000 \\
\hline 5 & 1.0000 & 1.0000 & 0.9876 & 0.5418 & 0.0530 & 0.5494 & 0.9894 & 1.0000 & 1.0000 \\
\hline 6 & 1.0000 & 1.0000 & 0.9820 & 0.4582 & 0.0340 & 0.4738 & 0.9826 & 1.0000 & 1.0000 \\
\hline 7 & 1.0000 & 1.0000 & 0.9820 & 0.5418 & 0.0530 & 0.5494 & 0.9826 & 1.0000 & 1.0000 \\
\hline 8 & 1.0000 & 1.0000 & 0.9722 & 0.4582 & 0.0340 & 0.4738 & 0.9720 & 1.0000 & 1.0000 \\
\hline 9 & 1.0000 & 1.0000 & 0.9876 & 0.5420 & 0.0530 & 0.5496 & 0.9894 & 1.0000 & 1.0000 \\
\hline 10 & 1.0000 & 1.0000 & 0.9820 & 0.4582 & 0.0340 & 0.4738 & 0.9826 & 1.0000 & 1.0000 \\
\hline 11 & 1.0000 & 1.0000 & 0.9820 & 0.5418 & 0.0530 & 0.5494 & 0.9826 & 1.0000 & 1.0000 \\
\hline 12 & 1.0000 & 1.0000 & 0.9820 & 0.4582 & 0.0340 & 0.4738 & 0.9826 & 1.0000 & 1.0000 \\
\hline 13 & 1.0000 & 1.0000 & 0.9820 & 0.4582 & 0.0340 & 0.4738 & 0.9826 & 1.0000 & 1.0000 \\
\hline 14 & 1.0000 & 1.0000 & 0.9820 & 0.5418 & 0.0530 & 0.5494 & 0.9826 & 1.0000 & 1.0000 \\
\hline
\end{tabular}


Am. J. Biostatistics 1 (2): 82-93, 2010

Table 7: The confidence intervals for all 14 methods for impaired fasting glucose data

\begin{tabular}{llllllll}
\hline Methods & 1 & 2 & 3 & 4 & 5 & 6 & 7 \\
\hline Upper & 0.097 & 0.096 & 0.096 & 0.098 & 0.090 & 0.092 & 0.096 \\
Lower & 0.038 & 0.041 & 0.041 & 0.039 & 0.036 & 0.034 & 0.041 \\
Length & 0.058 & 0.056 & 0.056 & 0.059 & 0.055 & 0.058 & 0.055 \\
Methods & 8 & 9 & 10 & 11 & 12 & 13 & 14 \\
Upper & 0.098 & 0.093 & 0.095 & 0.093 & 0.095 & 0.096 & 0.097 \\
Lower & 0.039 & 0.037 & 0.035 & 0.038 & 0.037 & 0.038 & 0.040 \\
Length & 0.058 & 0.056 & 0.060 & 0.055 & 0.058 & 0.058 & 0.057 \\
\hline
\end{tabular}

Table 8: The confidence intervals for all 14 methods for ethnic Fijian Women data

\begin{tabular}{llllllll}
\hline Methods & 1 & 2 & 3 & 4 & 5 & 6 & 7 \\
\hline Upper & 0.337 & 0.331 & 0.330 & 0.341 & 0.311 & 0.321 & 0.332 \\
Lower & 0.100 & 0.113 & 0.112 & 0.105 & 0.089 & 0.079 & 0.111 \\
Length & 0.237 & 0.218 & 0.218 & 0.236 & 0.222 & 0.242 & 0.222 \\
Methods & 8 & 9 & 10 & 11 & 12 & 13 & 14 \\
Upper & 0.342 & 0.320 & 0.330 & 0.321 & 0.333 & 0.337 & 0.333 \\
Lower & 0.101 & 0.100 & 0.090 & 0.102 & 0.094 & 0.098 & 0.111 \\
Length & 0.242 & 0.220 & 0.240 & 0.219 & 0.238 & 0.239 & 0.222 \\
\hline
\end{tabular}

The researchers found that five of the respondents reported at least weekly episodes of binge eating during the previous 6 months. We would like to test the proportion of Fijian women engaged in at least weekly episodes of binge eating is different from 0.20 . From the data we have $\hat{p}=5 / 50=0.10$. The results of the test from all the methods discussed here are given in Table 8. As in the previous example, all methods leads to the same conclusion of do not reject $\mathrm{H}_{0}$, at the significance level 0.05 . However, we might consider tests those have high power and size of test is close to nominal size. It should be noted that methods which showed significance level larger than specified $\alpha=0.05$ in simulation have shorter length in the examples. One should be careful in making decision based on these methods that have shorter length because of higher chance of Type I error.

\section{CONCLUSION}

In this study we considered various available test statistics for testing the proportion of a binomial distribution. Since a theoretical comparison is not possible, a simulation study has been made to compare the performance of the test statistics in the sense of attaining nominal size and power of the test. Our simulation indicate that among proposed test statistics, Method 6, Method 8, Method 10 and Method 12 performed better compared to the rest. However, Methods 1, 4, 6, 8, 10, 12 and 13 are promising and can be recommended to the practitioners. However, the practitioners should be careful about the hypothesized value of the binomial parameter $\mathrm{p}$. If the hypothesized value is near 0.5 , any test is acceptable for moderate to large sample size. However, for testing the end or small value of $p$, one might need very large sample size to have a good power and actual size of the test. Two real life data are analyzed to illustrate the proposed test statistics of the study.

\section{ACKNOWLEDGMENT}

Authors are thankful to the editor for his valuable comments which certainly improved the qulaity and presentation of the paper. This study was written while the second author was on sabbatical leave (2010-2011). He is grateful to Florida International University for awarding him the sabbatical leave which, gave him excellent research facilities.

\section{REFERENCES}

Agresti, A. and B.A. Coull, 1998. Approximate is better than "Exact" for interval estimation of binomial proportions. Am. Statis., 52: 119-126.

Anscombe, F.J., 1948. The transformation of Poisson, Binomial and negative-binomial data. Biometrika, 35: 246-254.

Barker, L., 2002. A comparison of nine confidence intervals for a Poisson parameter when the Expected number of events is $\leq 5$. Am. Statis., 56: 85-89.

Bartlett, M.S., 1947. The use of transformations. Biometrics, 3: 39-52.

Becker, A.E, Burwell, R.A., K. Navara and Gilman S.E., 2003. Binge Eating and Binge Eating Disorder in a small-scale, Indigenous society: The view from Fiji. Int. J. Eat. Disorders, 34: 423-431. DOI: $10.1002 /$ eat. 10225

Brown, L.D, T.T. Cai and A. DasGupta, 2001. Interval Estimation of a Binomial Proportion. Stat. Sci., 16: 101-133.

Cai, T.T., 2005. One-sided confidence intervals in discrete distributions. J. Statis. Plann. Inference, 131: 63-68. DOI: 10.1016/j.jspi.2004.01.005

Casella, G., 1986. Refining binomial confidence intervals. Canadian J. Statis., 14: 113-129. DOI: $10.2307 / 3314658$

Clopper, C.J. and E.S. Pearson, 1934. The use of confidence or fiducial Limits illustrated in the case of the Binomial. Biometrika, 26: 404-413. DOI: 10.1093/biomet/26.4.404

Efron, B., 1987. Better bootstrap confidence intervals. J. Am. Statis. Assoc., 82: 171-185. 
Flowerdew, R. and M. Aitkin, 2006. A method of fitting the gravity model based on the poisson distribution. J. Regional Sci., 22: 191-202. DOI: $10.1111 /$ j.1467-9787.1982.tb00744.x

Freeman, M.F. and J.W. Tukey, 1950. Transformations related to the angular and the square root. Annal. Math. Statis., 21: 607-611.

Garwood, F., 1936. Fiducial Limits for the poisson distribution. Biometrika, 28: 437-442. DOI: 10.1093/biomet/28.3-4.437
Wagenknecht, L.E., C.D. Langefeld, A.L. Scherzinger, J.M. Norris and S.M. Haffner et al., 2003. Insulin sensitivity, insulin secretion, and abdominal fat: The insulin resistance atherosclerosis study (Iras) family study. Diabetes, 53: 2490-2496. DOI: 10.2337/diabetes.52.10.2490

Wilson, E.B., 1927. Probable inference, the law of succession, and statistical inference. J. Am. Statis. Assoc., 22: 209-212. 